\title{
A Magnetic Resonance Measurement Technique for Rapidly Switched Gradient Magnetic Fields in a Magnetic Resonance Tomograph
}

\author{
K. Bartušek, E. Gescheidtová
}

This paper describes a method for measuring of the gradient magnetic field in Nuclear Magnetic Resonance (NMR) tomography, which is one of the modern medical diagnostic methods. A very important prerequisite for high quality imaging is a gradient magnetic field in the instrument with exactly defined properties. Nuclear magnetic resonance enables us to measure the pulse gradient magnetic field characteristics with high accuracy. These interesting precise methods were designed, realised, and tested at the Institute of Scientific Instruments (ISI) of the Academy of Sciences of the Czech Republic. The first of them was the Instantaneous Frequency (IF) method, which was developed into the Instantaneous Frequency of Spin Echo (IFSE) and the Instantaneous Frequency of Spin Echo Series (IFSES) methods. The above named methods are described in this paper and their a comparison is also presented.

Keywords: nuclear magnetic resonance, gradient magnetic field, magnetic resonance tomography, instantaneous frequency methods, spin echo.

\section{Notation}

$B_{0}(t) \quad$ Induction of the basic magnetic field

$G(t) \quad$ Gradient of the magnetic field

$G_{\alpha}\left(t^{\prime}\right)$ Gradient of the magnetic field in the $\alpha$ direction ( $\alpha$ is the $x, y$, or $z$ direction)

$M_{\mathrm{T}}(t)$ Macroscopic vector of the magnetisation of the nuclei

$m \quad$ Vector of the spatial nucleus magnetisation

$\gamma \quad$ Gyromagnetic ratio of the nucleus

$s(t) \quad$ MR signal

$s(n T)$ Digitalised MR signal

$f(t) \quad$ Instantaneous frequency of MR signal

$\Phi(t) \quad$ Instantaneous phase of MR signal

$T_{2} \quad$ Spin relaxation time

$T_{\mathrm{E}} \quad$ Echo time - time of the spin echo creation

\section{Introduction}

The quality of the gradient magnetic fields is an important property of devices exploiting the phenomenon of magnetic resonance for imaging or localised spectroscopy. Besides the homogeneity of the gradient field causing shape distortion of the MR image, the behaviour of the magnetic field during its time changes is also important. The time characteristics of the magnetic field in the instrument influence the amplitude of the scanned MR signal and the prolongation of the scanning time of the MR image.

Gradient changes induce eddy currents in nearby conducting arrangement, potentially causing image artefact, localization errors, and signal distortion. While the use of actively shielded gradients has greatly reduced the magnitude of eddy currents, significant distortion often still remains especially in short time interval after gradient switch off. Residual eddy currents may require further reduction. This is frequently achieved by preemphasis correction in relevant gradient canal and in the homogeneous $B_{0}$ shim. Rise time of the impulse of a magnetic field or its decrease to the level of non-homogeneity of a basic magnetic field should be as short as possible $(<100 \mu \mathrm{s})$.

Settings of the amplitude and time constants for preemphasis correction are based on the iterative process until eddy currents effects are minimized. This process uses one or more of the following measurement methods: output of a pick up coil, which needs special hardware and repositioning of the pick up coil [1, 2]; measuring multiple FIDs of a sample after a gradient is switch off, which needs precise sample positioning [3, 4]; mapping eddy current characteristics in the magnet for a small sample, which is a time-consuming procedure; or mapping along projections and adjusting the parameters for eddy current compensation by the automatic shimming technique $[5,6]$, which in measurement process uses all three gradients and doesn't allow measured for short time after the end of the gradient [7].

The basic idea of the MR gradient measurement method is to acquire the MR signal after selective excitation of a thin layer of the specimen and after the end of the gradient pulse [8]. At this point, the time gradient characteristic is proportional to the instantaneous frequency of the MR signal, which has a small signal to noise $(\mathrm{S} / \mathrm{N})$ ratio.

The time magnetic field induction characteristic in a limited layer is proportional to the instantaneous frequency of the complex MR signal, which is the sum of the FID signal originated after the application of the $\pi$ impulse and spin echo

$$
B\left(z_{0}, t\right)=\frac{\omega\left(z_{0}, t\right)}{\gamma} .
$$

In conductive parts of the MR device eddy currents induce, causing inconvenient retardation of the time changes of the magnetic field. The rise time of the impulse of the magnetic field or its decrease to the level of non-homogeneity of the basic magnetic field should be as short as possible $(<100 \mu \mathrm{s})$. The influence of eddy currents can be eliminated 
by an inverse filter, put into the way of the signals determining the time sequence of the generated gradient pulses. The constants of the digital inverse filters, so called preemphasis constants, are computed from the time courses of the disappearing gradient magnetic fields. These time characteristics must be measured very exactly for a sufficiently long time. If this condition is not fulfilled, significant errors are introduced during the calculations of the preemphasis constants and the compensation of the eddy currents is not sufficient. For this reason we try to measure the drop of the magnetic fields for as long a time period as possible.

An accurate method that is appropriate for time characteristics measurement is a method based on NMR, called the Instantaneous Frequency method [8]. The measurement can be performed on a commercial NMR instrument with adjusted preemphasis compensation. This method can be used to find out the quality of the gradient magnetic fields with sufficient precision. The disadvantage of the IF method lies in the very limited time interval - about to $2.5 \mathrm{~ms}$ - in which we are able to acquire an MR signal for further processing. For this reason the basic IF method is extended with spin echo, and is thus converted into the Instantaneous Frequency of Spin Echo (IFSE) method. The spin echo was created by using a $\pi$ exciting pulse. The IFSE method enables scanning of the MR signal for an $18 \mathrm{~ms}$ time period. The latest modification of the methods based on instantaneous frequency measurement is the Instantaneous Frequency of Spin Echoes Series (IFSES) method, which partially eliminates the main disadvantage of the two methods mentioned above. Its basic principle is the same as for the IFSE method [9]. The difference is that IFSES is based on the sum of the MR signals with echoes, measured at different echo times in order to extend the MR signal scanning time up to $80 \mathrm{~ms}$.

\section{IF method}

Direct measurement of the magnetic field gradients in the whole space of the tomograph is not possible because the MR signal, called the Free Induction Decay (FID) signal, carrier of the information about the gradient field time characteristics, decays rapidly $(100 \mu \mathrm{s})$. The FID signal is a complex signal, whose magnitude rapidly decreases, especially in the first part of the time domain. This effect is a consequence of rapid MR signal dephasing under the presence of a high gradient amplitude.

The gradients are computed from the amplitudes of the magnetic field induction in a thin layer of the investigated material, placed at a distance $\pm z_{0}$ from the centre of the gradients. In this case the FID signal lasts for a significantly longer time period. The location of a thin excited layer is determined by offset of the selective radio frequency pulse applied before the end of the gradient rectangular pulse. The pulse sequence of the IF method is shown in Fig. 1. At first the gradient pulse of $2 \mathrm{~s}$ length is initiated on the basis of the selected direction. After stabilisation of the eddy currents in the conductive parts of the MR magnet the nuclei are excited in the presence of the gradient by the selective $\pi / 2$ radio frequency (RF) pulse of $1.8 \mathrm{~ms}$ length. By adjusting the exciting coils current the basic magnetic field induction $B_{0}$ is set up to its maximum homogeneity. The zero gradient $B_{0}(t)$ is given by the sum of inductions of the magnetic field in positions $+z_{0}$

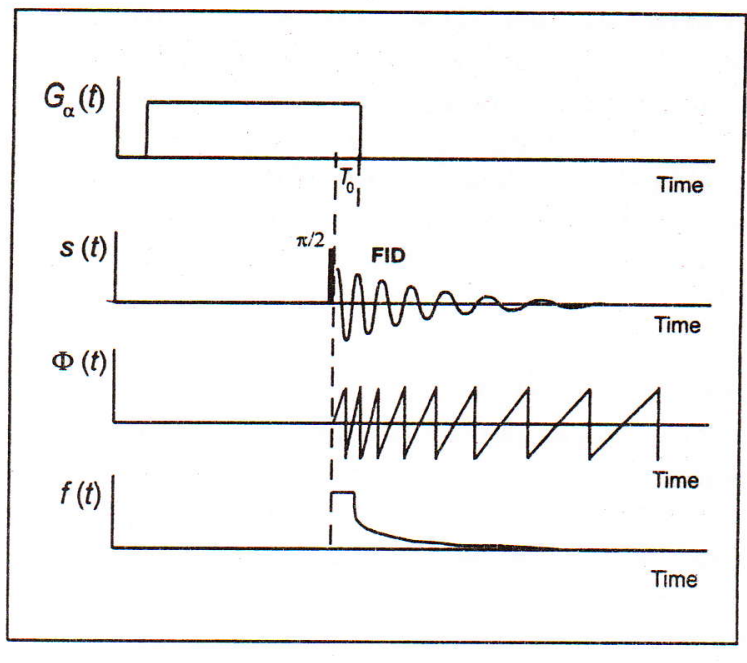

Fig. 1: Pulse sequence for the IF method

and $-z_{0}$, and the difference of the two parts determines the module of the magnetic field gradient.

$$
\begin{aligned}
& B_{0}(t)=\frac{1}{2}\left[B\left(z_{0}, t\right)+B\left(-z_{0}, t\right)\right], \\
& G_{\alpha}(t)=\frac{1}{2 z_{0}}\left[B\left(z_{0}, t\right)-B\left(-z_{0}, t\right)\right] .
\end{aligned}
$$

The local magnetic field is proportional to the instantaneous frequency of the FID signal; the frequency can be computed as a time derivative of the digitalised FID signal phase. During the digitalisation of the FID signal, the Shannon theorem has to be fulfilled. The digitalised FID signal is:

$$
s(n T)=\operatorname{Re}(s(n T))+j \operatorname{Im}(s(n T)) .
$$

The instantaneous phase of $s(n T)$ is:

$$
\Phi(t)=\operatorname{arctg}\left(\frac{\operatorname{Im}(s(n T))}{\operatorname{Re}(s(n T))}\right) .
$$

The instantaneous frequency is given by the time derivative of $s(n T)$ :

$$
\omega(t)=\frac{\mathrm{d}}{\mathrm{d} t} \Phi(t) \approx \frac{\Phi(t)-\Phi(t-T)}{T} .
$$

The gradient $G_{\alpha}(t)$, in the axis $\alpha$ direction, and induction $B_{0}(t)$ are computed using equations (2) and (3). The requirement is that the frequency of nuclei without the gradient pulse influence has to be set up into resonance.

The process of instantaneous frequency measurement is implied in the block diagram, Fig. 2. The FID signal is at first processed by an anti-aliasing filter (low-pass filter), followed by the $\mathrm{A} / \mathrm{D}$ converter. The digitalised FID signal $s(n T)$ is filtered by two digital filters. Between these two filters the instantaneous frequency computation block (IFC) takes place. The first filter (DF1) processes the FID signal in the time domain, the second (DF2) in the instantaneous frequency domain.

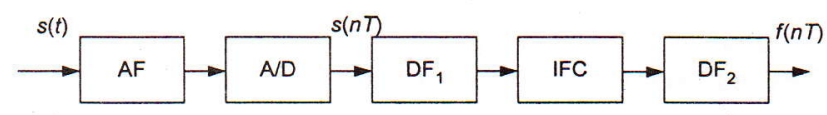

Fig. 2: Block diagram for the IF method 
Filtering a noised FID signal with variable frequency using classical digital filters is a big problem; particularly in the second part in the time domain, the FID signal has a very small $\mathrm{S} / \mathrm{N}$ ratio. The output signal has a large distortion, which is caused by the transient response of the common FIR filters used [10]; the error of measurement is too large. This disadvantage can be removed by the adaptive digital filtering method.

\section{IFSE method}

The principle of the IFSE method is identical to the IF method. The pulse sequence of the IFSE method is illustrated in Fig. 3. First a gradient pulse of $2 \mathrm{~s}$ length is initiated based on the selected direction. After stabilisation of the eddy currents in the conductive parts of the MR magnet the nuclei are excited in the presence of the gradient by the selective $\pi / 2$ high frequency pulse of $1.8 \mathrm{~ms}$ length. After time TE ( $4 \mathrm{~ms}$ ) the nuclei subside and FID quickly drops. The second selected pulse $\pi$ (3.6 ms) turns the direction of the nuclei rotation and after a certain time spin echo will be created.

The MR signal obtained during the measurement is consequently processed in the MATLAB environment. The instantaneous frequency is determined from the time deriva-

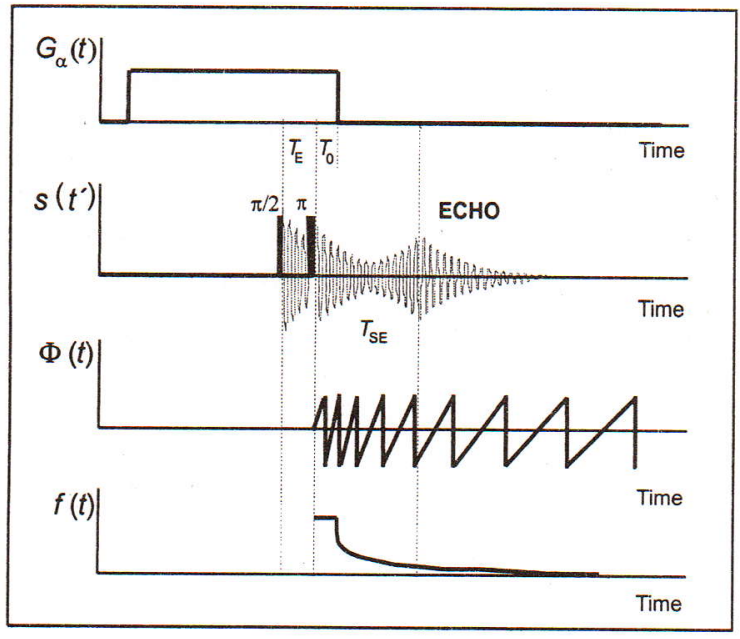

Fig. 3: Pulse sequence for the IFSE method

tion of the phase of the digitalised complex MR signal [1]. The total of the magnetisation vectors $m$ of particular excited nuclei in the whole excited space is represented by an integral. In our case the excited space is a thin circle layer placed in location $x_{0}$ and in coordinate $y$. The spin echo arises in the location $t^{\prime}=T_{\mathrm{E}}$. We measure signal $s\left(t^{\prime}\right)$ and for one echo it is:

$s\left(t^{\prime}\right)=\iint_{\text {slice }} m \cdot e^{-\frac{t^{\prime}}{T_{2}}} \cdot \exp \left[-j \int_{\frac{T_{\mathrm{E} i}}{2}}^{t^{\prime}} \cdot x \cdot G_{x}\left(t^{\prime}\right) \mathrm{d} t\right] \mathrm{d} x \mathrm{~d} y$.

The centre of the spin echo occurs in time $T_{\mathrm{SE}}(10 \mathrm{~ms})$. The spin echo is not symmetrical and does not have a maximum in $T_{\mathrm{E}}$ after termination of pulse $\pi$, as in the case in classical MR spectroscopy. This is caused by the dropping amplitude of the gradient magnetic field. We have to use a sample with a single thin spectral line to achieve sufficient precision of the measurement.

The time gradient characteristic is proportional to the instantaneous frequency of the complex MR signal. The MR signal used for later processing is the sum of the FID signal after the $\pi$ pulse and the spin echo. For consecutive MR signal processing it is important that the spin echo continually extends the FID signal and together they form a continuous signal.

The shape and position of the envelope of the spin echo is not important for the process of measuring the magnetic field decay. It is important that the MR signal with sufficient signal to noise ratio lasts as long as possible and that the envelope does not pass zero. At intersection with zero, the phase changes by a step and results in undesired impulse errors in the sequence of the instantaneous frequency of the MR signal and in the measured time characteristic of the gradient magnetic field. The time characteristic of the decaying gradient magnetic field can be measured to a maximum of $18 \mathrm{~ms}$.

\section{IFSES method}

The IFSES method is a modification of the IFSE method and partially eliminates its main disadvantage. The basic principle of the IFSES method is the same as for the IFSE method [2]. The difference between these two methods is that IFSES is founded on the sum of the MR signals with echoes, measured at variable echo times $T_{\mathrm{E}}$ in order to extend the MR signal scanning time. By generating and adding of the spin echoes in different times we receive a continuous MR signal. The times $T_{\mathrm{E}}$ are chosen so that the individual echoes overlap each other. The pulse sequence of the IFSES method is presented in Fig. 4.

The shape and position of the spin echo envelope are not important for the measurement of a decrease in magnetic field. It is substantial that the MR signal with a high $\mathrm{S} / \mathrm{N}$ ratio

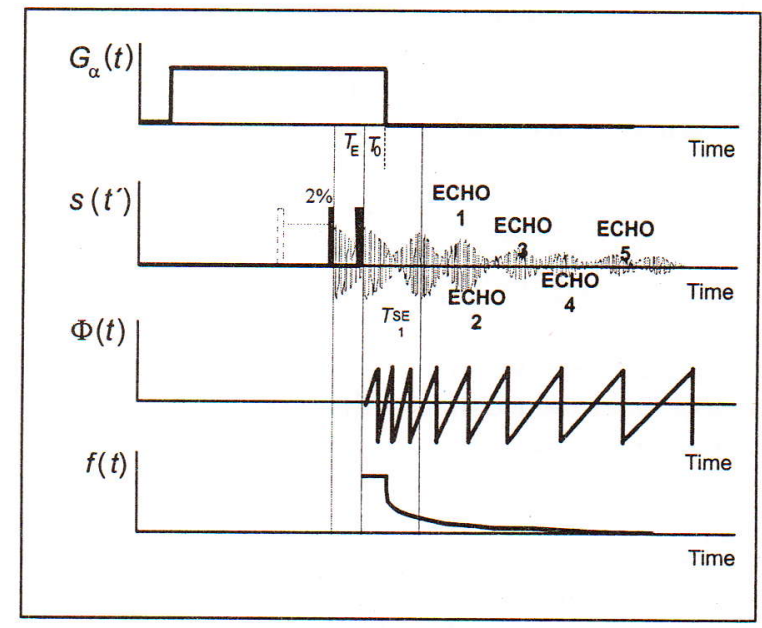

Fig. 4: Pulse sequence for the IFSES method

disappears for a sufficiently long time and its envelope does not go through zero. If the envelope passes through zero it results in a step change of the FID signal phase, and in an undesirable impulse error in the sequence of the instantaneous frequency. The computed time characteristics of the gradient magnetic field are not correct in this case. 
The time characteristics of a decreasing magnetic field in an ISI tomograph can be measured up to $80 \mathrm{~ms}$, when the S/N ratio of the FID signal is sufficiently high for consecutive processing. An example of an MR signal for a $G_{x}$ gradient is presented in Fig. 5. The MR signal obtained during the measurement is consequently processed in the MATLAB environment. The instantaneous frequency is determined from the time derivation of the phase of the digitalised complex MR signal [1].

The total MR signal of all echoes is:

$$
\sum s\left(t^{\prime}\right)=\sum_{i=T_{\mathrm{E} \text { min }}}^{T_{\mathrm{E} \max }} s\left(t^{\prime}\right) \text {. }
$$

We can write for total echo, using (7) and (8):

$$
\sum s\left(t^{\prime}\right)=\sum_{i=T_{\text {E min }}}^{T_{\text {Emax }}} \iint_{\text {slice }} m \cdot e^{-\frac{l^{\prime}}{T_{2}}} \cdot \exp \left[-j \int_{\substack{T_{E i} \\ 2}}^{\iota^{\prime}} \gamma \cdot x \cdot G_{x}\left(t^{\prime}\right) \mathrm{d} t\right] \mathrm{d} x \mathrm{~d} y \cdot(9)
$$

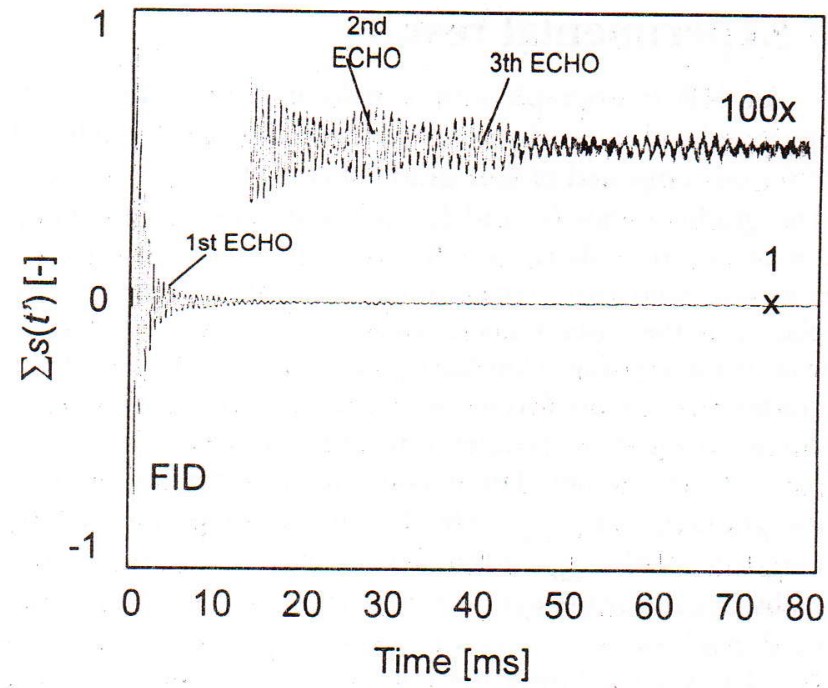

Fig. 5: Example of an MR signal for a $G_{x}$ gradient

IF METHOD

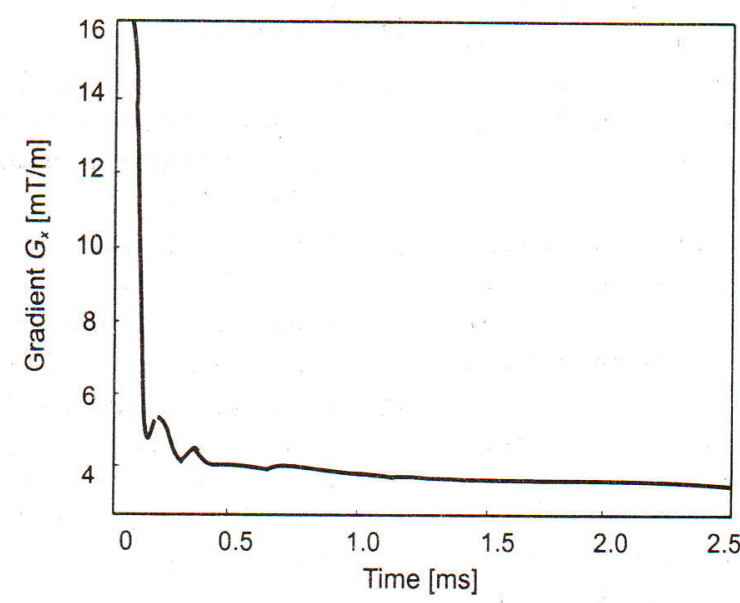

IFSE METHOD

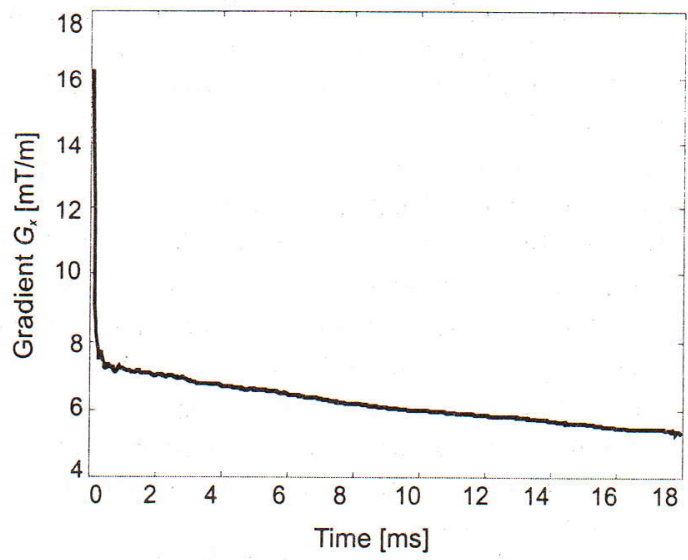

IFSES METHOD

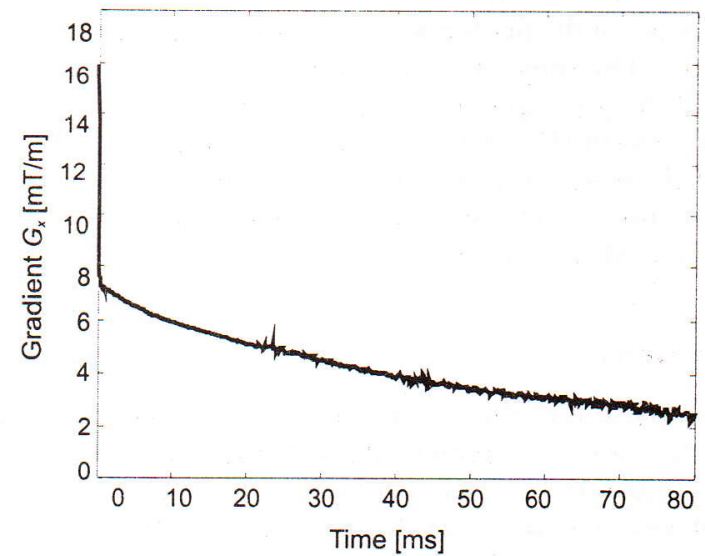

Fig. 6: Comparison of gradient $G_{x}$ decay time characteristics measured by IF, IFSE, and IFSES methods 


\section{Experimental results}

An MR tomograph with a field intensity of $4.7 \mathrm{~T}$ is equipped with a gradient system with an inner diameter of $200 \mathrm{~mm}$ composed of four gradient coils $\left(G_{x}, G_{y}, G_{z}\right.$ and $\left.B_{0}\right)$. The gradient coils $G_{x}$ and $G_{y}$ are constructed on a printed circuit board, coils $G_{z}$ and $B_{0}$ are cylindrical and they are wound around with a copper conductor. All gradient coils are placed in the instantaneous vicinity of the non-conductive wall of the cryostat. Conductive layers in the vicinity of the gradient system are formed with a skeleton of the supra-conductive magnet, its temperature-shielding layers and other parts of the magnet. The maximum current going through the gradient coils, $I_{\max }= \pm 60 \mathrm{~A}$ induces the gradient of the magnetic field $G_{\max }= \pm 100 \mathrm{mT} / \mathrm{m}$. The measurement was realised at the gradient $G_{\alpha}= \pm 20 \mathrm{mT} / \mathrm{m}$ ( $\alpha$ is a direction of the used gradient $-x, y$ or $z$ ). The pulse sequence presented in Fig. 4 was used during the measurement. A thin layer was excited by a rectangular $\pi / 2 \mathrm{RF}$ pulse. For a gradient at the amplitude of $20 \mathrm{mT} / \mathrm{m}$ the thickness of the excited layer is $0.94 \mathrm{~mm}$. Reversing the RF $\pi$ pulse will create a spin echo, and the MR signal is formed by resonating nuclei in the layer $0.47 \mathrm{~mm}$ in thickness. For offset of $6000 \mathrm{~Hz}$ and a gradient of $20 \mathrm{mT} / \mathrm{m}$ the excited layer was at a distance of $9.4 \mathrm{~mm}$ from the centre of the measured specimen, formed with a sealed glass ball with a diameter of $36 \mathrm{~mm}$, filled with distilled water. Homogeneity was achieved with tuning of the basic magnetic field $1 \cdot 10^{-7}$ on the ball with a diameter of $36 \mathrm{~mm}$. The MR signal was taken for $80 \mathrm{~ms}$, which is $100 \mu$ s before switching off the gradients. To prevent the influence of noise, the MR signal was accumulated five times.

The time characteristics of the decay times of the $G_{x}, G_{y}, G_{z}$ gradients of the magnetic field and the induction of the basic magnetic field $B_{0}$ were measured by the IFSES method in the working space of the tomograph without the assessment of preemphasis. Double adaptive filtration and averaging were used for the MR signal processing.

A comparison of the $G_{x}$ gradient time decay characteristics obtained by the IF, IFSE, and IFSES methods is presented in Fig. 6 . From the courses of the curves, it is obvious that all three methods give the same results in the interval up to $2.5 \mathrm{~ms}$ including systematic errors of measurement. The IFSES method gives the same results in the time interval of up to $18 \mathrm{~ms}$ as the IFSE method, but it enables measurement of time characteristics of the fields even $80 \mathrm{~ms}$ after the gradient pulse disappears. This interval is sufficiently long for accurate computation of the preemphasis constants. The time characteristic measured by the IFSES method is not complete; the $G_{x}$ gradient field decreases to a level of $29 \%$ in the course of $18 \mathrm{~ms}$ after the end of the pulse. The gradient declines to the inhomogeneity level up to $1 \mathrm{~s}$, or $2 \mathrm{~s}$.

\section{Conclusion}

From a comparison of the IF method and the two methods with spin echo for measurement of the gradient magnetic field time characteristics in MR systems, it is clear that the application of spin echo prolongs the interval when the time characteristics are measurable with sustained accuracy. A comparison of the IFSE and IFSES methods gives the same results, including systematic errors of measurement, in the interval to $18 \mathrm{~ms}$. From the results obtained by measurement it follows that both methods are convenient for simple and quick characterization of the gradient magnetic field in MR tomographic magnets.

The IFSES method prolongs the interval of gradient magnetic field time decay characteristics measurement four times more than the IFSE method. This prolongation is substantial for the computation of preemphasis constants and for their accuracy. An MR signal up to $80 \mathrm{~ms}$ after the end of a gradient has a sufficiently high $\mathrm{S} / \mathrm{N}$ ratio, and the results are sufficient for subsequent processing.

The described measurement techniques enabled exact adjustment of preemphasis constants in the MR system in the Institute of Scientific Instruments. This adjustment shows a significant sensibility to the exactness of the gradient fields course measurement. It was achieved, in comparison with other published techniques, the adjustment of preemphasis especially in the area up to $1 \mathrm{~ms}$ after the end of a gradient pulse.

\section{Acknowledgment}

This study has been supported by Grant No: A2065201 of the Grant Agency of the Academy of Sciences of the Czech Republic.

\section{References}

[1] Chankij, J., Lefevre, J. L., Briquet A.: A method for Mapping Magnetic Fields Generated by Current Coils. Journal of Physics E-scientific Instruments, Vol. 18, 1985, p. 1014-1017.

[2] Jensen, D. J., Brey, W. W., Delayre, J. L., Narayana, P. A.: Reduction of Pulsed Gradient Settling Time in the Super-conducting Magnet of a Magnetic-resonance Instrument. Med. Phys., Vol. 14, 1987, p. 859-862.

[3] Robertson, S., Hughles, D. G., Liu, Q., Allen P. S.: Analysis of the Temporal and Spatial Dependence of Eddy-current Fields in a 40-cm Bore Magnet. Magn. Reson. Med., Vol. 25, 1992, p. 158-166.

[4] Liu, Q., Hughes, D. G., Allen, P. S.: Quantitative Characterization of Eddy-current Fields in a Bore Superconducting Magnet. Magnet. Reson. Med., Vol. 31, 1994, p. 73-76.

[5] Hudges, D. G., Robertson, S., Allen, P. S.: Intensity Artefacts in MRI Caused by Gradient Switching in an Animal-size NMR Magnet. Magnet. Reson. Med., Vol. 25, 1992, p. 167-179.

[6] Liu, Y., Riederer, S. J., Ehman, R. L.: Magnetization-prepared Cardiac Imaging Using Gradient-echo Aquisition. Magnet. Reson. Med., Vol. 30, 1993, p. 271-275.

[7] Terpstra, M., Andersen, P. M., Gruetter, R.: Localized Eddy Current Compensation Using Quantitative Field Mapping. Magnet. Reson. Med., Vol. 131, 1998, p. 139-143.

[8] Bartušek, K.,Gescheidtová, E.: Exploitation of the Method of Instantaneous Frequency for Measurement of Time Characteristics of Gradient Magnetic Fields in MR Tomography. Elektrorevue, Vol. 26, 2002, http://www.elektrorevue.cz/obsah.html.

[9] Bartušek, K.,Gescheidtová, E.: Instantaneous Frequency of Spin Echo Method for Gradient Magnetic Fields Measurement 
in MR Systems. Journal of Electrical Engineering, Vol. 53, 2002, p. 49-52.

[10] Bartušek, K., Gescheidtová, E.: Adaptive Digital Filter for Gradient Magnetic Fülds Measurement in MR Tomograph. IEEE Conference APCCAS 2002, Singapore, ISBN 0-7803-7690-0, p. 79-82.

Ing. Karel Bartušek, DrSc.

e-mail: bar@isibrno.cz

Academy of Sciences of the Czech Republic

Institute of Scientific Instruments

Královopolská 147

61264 Brno, Czech Republic

Ing. Eva Gescheidtová, CSc.

e-mail: gescha@feec.vutbr.cz

Department of Theoretical and Experimental Electrical Engineering

Brno University of Technology

Faculty of Electrical Engineering and Communication

Purkyňova 118

61200 Brno, Czech Republic 